\title{
An Abundance Estimation Method Based on Matrix Factorization for Hyperspectral Images
}

\author{
Wei Xia ${ }^{1, a}$ \\ ${ }^{1}$ China Transport Telecommunications \& Information Center, Beijing 100011, China \\ aweixia@live.cn
}

Keywords: remote sensing imagery, hyperspectral unmixing, abundance estimation, matrix triangular factorization.

\begin{abstract}
For hyperspectral remote sensing imagery, the observation of image pixel usually consists of more than one material, causing it to be a "mixed pixel". In order to analyze the hyperspectral dataset, it is necessary to decompose the mixed pixels into a collection of substances' spectra and their corresponding abundance proportions. However, hyperspectral dataset usually contains hundreds of spectral images, which brings rather large computational complexity. This paper presents a quick approach to estimate the abundances by exploiting matrix triangular factorization, which can rectify possible bias in the given spectral by utilizing the data's geometric spatial information. This property is rather effective especially when no pure-pixel presents in the imagery. Experimental results on real hyperspectral data indicate that the proposed approach can obtain desirable results.
\end{abstract}

\section{Introduction}

In recent years, hyperspectral remote sensing has become an active field. Due to the limitation of spatial resolution, the observation of a pixel usually consists of more than one material, so it is necessary to decompose the "mixed pixels" into a collection of substances' spectra and their corresponding proportions. This process is called hyperspectral unmixing, which has become an important process for applications, such as monitoring, agriculture, military surveillance, etc. Most algorithms for hyperspectral unmixing are based on linear mixture model (LMM), which assumes that the observation of image signal is the linear mixture of spectra (called endmembers) weighted by their corresponding fractions (called abundances) [1]. The task of unmixing is algebraically equivalent to the task of matrix factorization. That is, decomposing the observation matrix into two nonnegative matrices. The two matrices are spectral matrix and abundance matrix, respectively.

The spectral matrix can be obtained by searching for the "pure pixels" as endmembers from the observation, i.e., find the vertices of a set of points in the L-dimensional geometry space formed by the hyperspectral dataset, where $L$ is the number of spectral bands [2]. Here, "pure pixel” means a pixel only consists of a single kind of material with $100 \%$ proportion in its image scene, with no mixture with other substances. The corresponding approach is named as endmember extraction [3]. Given the spectral matrix, the abundance matrix can be obtained by inferring the corresponding proportions associated with all the endmembers for each observation pixel. This approach is called as abundance estimation, and its accuracy depends on the given endmembers. Generally, it is assumed that at least one pure pixel presents for each material, and then the results of endmember extraction are accurate. Otherwise the obtained endmember spectra and the corresponding abundances may not be correct. In order to perform in the situation without pure pixel assumption, this paper presents an approach, which can determine the endmember and abundance signatures simultaneously. The method is designed by combining the idea of matrix triangular factorization with two LMM inherent characteristics, the Abundance Nonnegative Constraint (ANC) and Abundance Sum-to-one Constraint (ASC), both of which are essential attributes with clear physical meaning for hyperspectral imagery. The proposed approach not only performs with a fast speed, but also allows possible errors 
to exist in the given spectra. It rectifies the inaccurate endmembers and determines the abundance simultaneously.

\section{Related Work}

This section introduces the basic definitions and notations for the complex network topology and the notations involved.

Linear Mixture Model (LMM). In order to unmixing image, the LMM is applied, which assumes the observation of a pixel is a linear combination of all endmembers' spectral signatures weighted by their corresponding abundance fractions. Consider a hyperspectral data of $N$ distinct pixels over $L$ spectral bands, the observation signal of a single pixel $\mathbf{x} \in \mathbb{R}^{L \times 1}$ can be given as:

$$
\mathbf{x}=\mathbf{A s}+\mathbf{n} \text {. }
$$

Where $\mathbf{A}=\left[\mathbf{a}_{1}, \mathbf{a}_{2}, \ldots, \mathbf{a}_{P}\right] \in \mathbb{R}^{L \times P}$ is the spectral matrix, with its $i$ th column $\mathbf{a}_{i} \in \mathbb{R}^{L \times 1}$ representing the spectrum of the $i$ th endmember. $P$ is the endmembers number. $\mathbf{s}=\left[s_{1}, s_{2}, \ldots, s_{p}\right]^{\mathrm{T}} \in \mathbb{R}^{P \times 1}$ represents the abundance. $\mathbf{n} \in \mathbb{R}^{L \times 1}$ is the error vector. $\mathbf{s}$ is subjected to two constraints, ANC and ASC, which means that the percentages of all endmembers in any pixel must be nonnegative and sum to one:

$$
0 \leq s_{i}, \quad \sum_{i=1}^{P} s_{i}=1, \quad(i=1,2, \ldots, P) .
$$

For a hyperspectral scene of $N$ distinct pixels for each image band, the observation vectors can be arranged as the columns of an observation matrix $\mathbf{X} \in \mathbb{R}^{L \times N}$, and equation (1) is written as

$$
\mathbf{X}=\mathbf{A S}+\mathbf{N} \text {. }
$$

Here, each column of $\mathbf{X}$ represents the observation of a pixel, and each row of abundance matrix $\mathbf{S} \in \mathbb{R}^{P \times N}$ is the abundance distribution of an endmember. $\mathbf{N}$ is the related error matrix.

The Matrix Factorization (MF) . The MF decomposes a matrix into a product of two matrices. There are different MF methods, in this paper, we use the QR decomposition, given by $\mathbf{H}=\mathbf{Q R}$, where $\mathbf{Q}$ is an orthogonal matrix of size $\mathrm{m}$-by-m, and $\mathbf{R}$ is an upper triangular matrix of size m-by-n. The full-size $\mathrm{QR}$ factorization produces a square, m-by-m orthogonal $\mathbf{Q}$ and a rectangular m-by-n upper triangular $\mathbf{R}$. The last $(m-n)$ rows of $\mathbf{R}$ are all equal 0 , which are not needed in most cases. Hence, in practice only the first $\mathrm{n}$ columns of $\mathbf{Q}$ and the first $\mathbf{n}$ rows of $\mathbf{R}$ are computed. This simplified QR factorization is called economy-size QR factorization, producing a rectangular, m-by-n $\mathbf{Q}$ with orthonormal columns and a square, n-by-n upper triangular $\mathbf{R}$.

\section{The proposed method}

Given the observation matrix $\mathbf{X}$ and the obtained spectral matrix A, Decompose the spectral matrix A by $Q R$ factorization $\mathbf{A}=\mathbf{Q R}$, then the linear mixture model (1) can be given as $\mathbf{X}=\mathbf{Q R s}$. Note that the error vector $\mathbf{n}$ in (1) is omitted for ease of presentation. Since $\mathbf{Q}^{\mathrm{T}} \mathbf{Q}=\mathbf{I}$ (I is a vector with all its elements is one), we can transform equation $\mathbf{x}=\mathbf{Q R s}$ into $\mathbf{Q}^{\mathrm{T}} \mathbf{x}=\mathbf{R s}$, which can be written as

$$
\left[\begin{array}{l}
b_{1} \\
b_{2} \\
\vdots \\
b_{P}
\end{array}\right]=\mathbf{Q}^{\mathrm{T}}\left[\begin{array}{l}
x_{1} \\
x_{2} \\
\vdots \\
x_{L}
\end{array}\right]=\left[\begin{array}{ccccc}
r_{11} & r_{12} & \cdots & r_{1(P-1)} & r_{1 P} \\
0 & r_{22} & \cdots & r_{2(P-1)} & r_{2 P} \\
0 & 0 & \ddots & \vdots & \vdots \\
\vdots & \vdots & \ddots & r_{(P-1)(P-1)} & r_{(P-1) P} \\
0 & 0 & \cdots & 0 & r_{P P}
\end{array}\right]\left[\begin{array}{l}
s_{1} \\
s_{2} \\
\vdots \\
s_{P-1} \\
s_{P}
\end{array}\right]
$$

Here $\mathbf{x}, \mathbf{Q}$ and $\mathbf{R}$ are known quantities ( $\mathbf{Q}$ and $\mathbf{R}$ can be obtained by spectral matrix $\mathbf{A}$ ), i.e. $b_{i},(i=1,2, \ldots, P)$ is known. The unknown abundance $s_{i},(i=1,2, \ldots, P)$ can be obtained by solving linear simultaneous equation (4). Matrix $\mathbf{R}$ is a lower triangular matrix, so the calculation of $s_{i}$ is very easy and there is no need to calculate matrix inversion of $\mathbf{R}$. The resulting formula of equation (4) is 


$$
\begin{aligned}
& s_{P}=\frac{b_{P}}{r_{p p}} \\
& s_{P-1}=\frac{b_{P-1}}{r_{P-1, P-1}} \\
& \vdots \\
& s_{1}=\frac{b_{1}-\sum_{i=2}^{P} r_{1 i} s_{i}}{r_{11}}
\end{aligned}
$$

Under the matrix form (3), the solving of abundance matrix can be written as $\mathbf{S}=\operatorname{inv}(\mathbf{R}) \mathbf{Q}^{\mathrm{T}} \mathbf{X}$. Equation (5) indicates that the matrix inversion is not necessary for estimating $s_{i}$, hence equation $\mathbf{S}=\operatorname{inv}(\mathbf{R}) \mathbf{Q}^{\mathrm{T}} \mathbf{X}$ just represents the process of obtaining abundance by solving equation (4), and the symbol $\operatorname{inv}(\mathbf{R})$ is not necessary to be the matrix inversion of $\mathbf{R}$.

The above analysis uses matrix $\mathbf{A}$ and $\mathbf{X}$ to solve the linear system $\mathbf{X}=\mathbf{A S}$. If transpose $\mathbf{X}=\mathbf{A S}$ to be: $\mathbf{X}^{\mathrm{T}}=\mathbf{S}^{\mathrm{T}} \mathbf{A}^{\mathrm{T}}$, so similarly one can obtain $\mathbf{A}^{\mathrm{T}}$ by solving the linear system $\mathbf{X}^{\mathrm{T}}=\mathrm{S}^{\mathrm{T}} \mathbf{A}^{\mathrm{T}}$ if $\mathbf{X}^{\mathrm{T}}$ and $\mathbf{S}^{\mathrm{T}}$ are known. Decompose $\mathbf{S}^{\mathrm{T}}=\mathbf{Q}_{\mathrm{S}} \mathbf{R}_{\mathrm{S}}$, then $\mathbf{A}$ can be calculated by $\mathbf{A}=\left(\operatorname{inv}\left(\mathbf{R}_{\mathrm{S}}\right) \mathbf{Q}_{\mathrm{S}}^{\mathrm{T}} \mathbf{X}^{\mathrm{T}}\right)^{\mathrm{T}}$.

The procedure of the proposed Abundance Quantification algorithm is described as follows:

1) Intput the hyperspectral images and the origanl spectral matrix, arrange:

$$
\mathbf{X}_{1}=\left[\begin{array}{l}
\mathbf{X} \\
\mathbf{1}^{\mathrm{T}}
\end{array}\right], \mathbf{A}_{1}=\left[\begin{array}{l}
\mathbf{A} \\
\mathbf{1}^{\mathrm{T}}
\end{array}\right] \text {, where } \mathbf{1}^{\mathrm{T}}=[1,1, \ldots, 1] \in \mathbb{R}^{1 \times P} .
$$

2) Decompose $\mathbf{A}_{1}=\mathbf{Q}_{\mathrm{A}} \mathbf{R}_{\mathrm{A}}$, and calculate $\mathbf{S}=\operatorname{inv}\left(\mathbf{R}_{\mathrm{A}}\right) \mathbf{Q}_{\mathrm{A}}^{\mathrm{T}} \mathbf{X}_{1}$

3) Find the elements which do not satisfy ASC in matrix $\mathbf{S}$, set

$$
m_{i j}=\left\{\begin{array}{ll}
s_{i j}, & \text { if } \sum_{i} s_{i j} \neq 1 \\
0, & \text { if } \sum_{i} s_{i j}=1
\end{array} .\right.
$$

Stop the algorithm if $\sum_{j} \sum_{i} m_{i j} / N / P<\zeta$, and output abundance $\mathbf{S}$ and the rectified spectral $\mathbf{A}$. Here $\zeta$ is a small value called error tolerance.

4) Scale and normalize the abundances matrix $S$

a) Scale every element of matrix $S$ to $[0,1]$ by

$$
s_{i j}=\left\{\begin{array}{ll}
1, & \text { if } s_{i j}>1 \\
0, & \text { if } s_{i j}<0
\end{array},(i=1,2, \ldots, P, j=1,2, \ldots, N)\right.
$$

b) Normalize $\mathbf{s}_{j}=\left[s_{1 j}, s_{2 j}, \ldots, s_{P j}\right]^{\mathrm{T}}$ by

$$
\tilde{\mathbf{s}}_{j}=\frac{\mathbf{s}_{j}}{\sum_{i=1}^{P} s_{i j}}, j=1, \ldots, N \text {. }
$$

5) Set $\tilde{\mathbf{S}}=\left[\tilde{\mathbf{s}}_{1}, \tilde{\mathbf{s}}_{2}, \ldots, \tilde{\mathbf{s}}_{P}\right]^{\mathrm{T}}$,perform QR decomposition $\tilde{\mathbf{S}}=\mathbf{Q}_{\mathrm{S}} \mathbf{R}_{\mathrm{S}}$ and rectify $\mathbf{A}$ by

$$
\mathbf{A}=\lambda\left(\operatorname{inv}\left(\mathbf{R}_{\mathrm{S}}\right) \mathbf{Q}_{\mathrm{S}}^{\mathrm{T}} \mathbf{X}_{1}^{\mathrm{T}}\right)^{\mathrm{T}}+(1-\lambda) \mathbf{A}
$$

where $\lambda$ is forgetting factor, satisfying $0<\lambda<1$.

6) Return to 1) to update $\mathbf{A}_{1}$.

\section{Experiment Evaluation}

This section uses hyperspectral images to test the proposed algorithm. The dataset was obtained in the area of Lunar Lake, Northern Nye County, state of Nevada in July, 1998. This data has 224 spectral bands, covering the southern part of Lunar Lake. Before unmixing, the noisy or water-absorption bands are removed, remaining a total of 167 bands for the experiment. The spatial resolution is $20 \mathrm{~m}$, which means that there exist many mixed pixels and it is hard to identify the 
non-typical objects directly from observed images. For this experiment, a sub-scene with size of 161 $\times 110$ is selected from the original data, and figure 1 shows the corresponding false color image. According to prior information [6], the materials in this dataset mainly include cinders, playa lake, rhyolite, shade, vegetation and anomaly. Figure 2 illustrates the generated unmixing results, and it can be found that the results correspond with the ground truth very well.

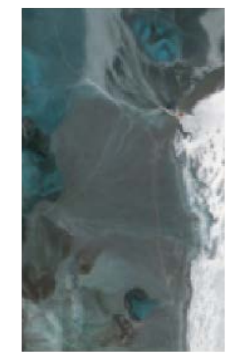

Fig. 1. The false color image.

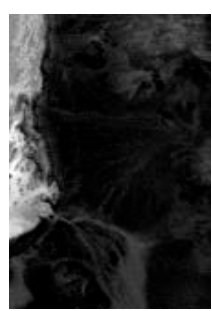

(a)

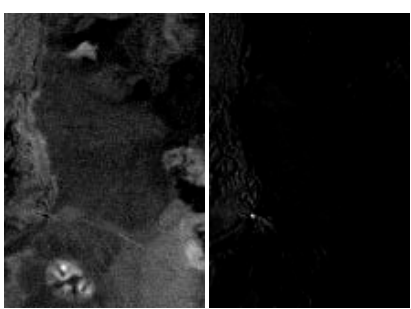

(b)

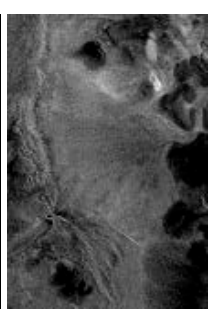

(d)

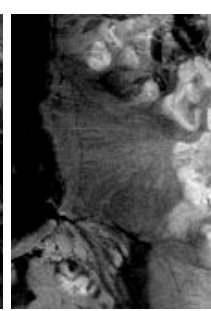

(e)

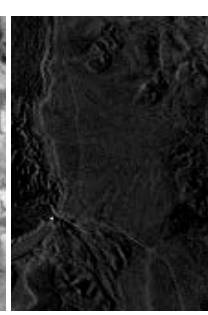

(f)

Fig. 2. Abundance images. (a) playa Lake, (b) cinder, (c) anomaly, (d) rhyolite, (e) vegetation, (f) shade.

\section{Summary}

This paper presents an approach for hyperspectral unmixing based on QR factorization, the method saves the computation cost by utilizing matrix triangular factorization, and it can rectify the biased spectra, further improving the unmixing accuracy. The method has both explicit physical meaning and simple operational process. Experimental results show that, as an algorithm with no need of pure pixel assumption, and this is useful for reducing the limitations caused by pure-pixel assumption.

\section{References}

[1] C.-I Chang: Hyperspectral Imaging: Techniques for Spectral Detection and Classification. New York: Plenum, 2003.

[2] J. Li and J. M. Bioucas-Dias: Minimum volume simplex analysis: a fast algorithm to unmix hyperspectral data. in Proc. IGARSS, Boston, MA, Vol.3 (2008), p. 250-253,.

[3] J. Nascimento and J. Bioucas-Dias: Vertex Component Analysis: A Fast Algorithm to Unmix Hyperspectral Data. IEEE Trans. Geosci. Remote Sens., Vol.43 (2002), p.898-910.

[4] Gene H. Golub, Charles F. Van Loan: Matrix Computations (3rd ed.), Johns Hopkins, 1996.

[5] L. Miao and H. Qi: Endmember extraction from highly mixed data using minimum volume constrained nonnegative matrix factorization. IEEE Trans. Geosci. Remote Sens., vol.45 (2007), p.765-777.

[6] S. Cai, Q. Du and R. Moorhead: Hyperspectral imagery visualization using double layers. IEEE Trans. Geosci. Remote Sens., vol. 45 (2007), p. 3028-3036. 